\title{
GÉNEROS DE LA COMUNICACIÓN INTERNACIONAL EN CURSOS DE SECRETARIADO EJECUTIVO
}

\section{GENRES OF INTERNATIONAL COMMUNICATION IN EXECUTIVE SECRETARIAL COURSES}

\section{Viviane Cristina Poletto Lugli}

Mestre em Estudos da Linguagem pela Universidade Estadual de Londrina - UEL

Professora de língua espanhola no Curso de Secretariado Executivo Trilíngue na Universidade Estadual de Maringá - UEM

E-mail: vivianelugli@yahoo.com.br (Brasil) 


\title{
SECRETARIADO EJECUTIVO
}

\section{RESUMÉN}

Este artículo tiene como objetivo demostrar la necesidad de la inserción de géneros textuales del ámbito internacional en clases de español de cursos de Secretariado Ejecutivo. En el intento de priorizar en el salón de clase un tipo de práctica social condicha con las situaciones de lenguaje constitutivas del contexto social y profesional de secretarios en formación, hicimos una investigación del tipo bibliográfica, a partir de la cual fueron analizados manuales didácticos destinados a la enseñanza de español empresarial para averiguar qué géneros de la comunicación internacional estaban presentes en los manuales de enseñanza. Teniendo como base un análisis referente a los tipos de géneros textuales, constatamos que algunos formularios que los secretarios ejecutivos necesitan conocer para ejecutar sus acciones laborales en empresas importadoras de productos del Mercosur no están vehiculados por los manuales didácticos analizados. Basándonos en esa carencia, seleccionamos los formularios "Carta de Porte" y el "Certificado de origen" para didactizarlos e intentar desarrollar en los alumnos las capacidades de lenguaje necesarias para trabajar con tales géneros en su ambiente profesional. Para la realización de esa investigación, nos guiamos por los fundamentos del interaccionismo socio-discursivo de la Universidad de Ginebra que postula la enseñanza a partir del enfoque en la lengua contextualizada y en la propuesta de trabajo con géneros textuales de un equipo de investigadores del Sur de Brasil (Cristovão, 2001;E.L.Nascimento, 2004, 2005, 2009).

Palabras-clave: Géneros; Secretariado; Libros.

\section{GENRES OF INTERNATIONAL COMMUNICATION IN EXECUTIVE SECRETARIAL COURSES}

\begin{abstract}
This work intends to show the necessity of insertion of genres of international ambit in Spanish classes in Executive Secretarial courses. With the objective of prioritizing in classroom a kind of social practice compatible with language situations which constitute social and professional contexts of secretaries, we performed a bibliographic research in which didactic books aimed to business Spanish teaching were analyzed in order to check which genres of international communication are mentioned. Based on an analysis concerning kinds of genres, we observed that some forms the executive secretaries should know in order to perform a job at Mercosul products importing companies are not found in the analyzed books. Given this need, we selected the forms "Transportation knowledge" and "Origin Certificate" to teach and try to develop language abilities required to work with such genres at professional environment. To that, we were guided by interactionism social discursive of Geneva University that settles that teaching should based on contextualized language and textual genres of a group of researchers in the south of Brazil (Cristovão, 2001; E.L.Nascimento, 2004, 2005, 2009).
\end{abstract}

Keywords: Genre; Secretarial; Books.

\section{INTRODUCCIÓN}

Revista de Gestão e Secretariado - GeSec, São Paulo, v. 4, n. 2, p 73-89, jul./dez. 2013. 
La articulación entre el objeto a enseñar y la herramienta de aprendizaje constituye un desafío en la enseñanza de lenguas extranjeras. Pese a que muchos materiales didácticos hayan sido producidos en los últimos años para la enseñanza de español para fines específicos, el docente, al iniciar un nuevo año didáctico, siempre se ve envuelto en la difícil tarea de tener que elegir uno de los materiales que les satisfazgan a las necesidades de aprendizaje de sus alumnos.

Ahora bien, si partimos del postulado de que son las prácticas de lenguaje significativas, socialmente reconocidas (Dolz \& Gagnon, 2010, p. 500) que deben orientar la enseñanza, necesitamos también volver la mirada para los materiales ya producidos en el mercado editorial con el fin de averiguar si la mejor opción es adoptarlos o implementar secuencias de trabajo en el aula producidas por el profesor.

Al implementar secuencias de trabajo, estaríamos desarrollando herramientas que según Marx (1868, p.182, apud Dolz \& Gagnon, 2010, p. 506) es uno de los trazos característicos del ser humano, que es un "animal fabricante de herramientas". Sin embargo, al referirse a este tipo de herramientas, Marx (op.cit) la concibe como herramienta cultural que se transmite de generaciones a generaciones.

Así, el ser humano, desde niño adquiere herramientas culturales en su proceso de formación y son estas herramientas que les sirven para comunicarse y desarrollarse en la vida social. Son herramientas que son transmitidas en la esfera familiar, en la esfera escolar, y que son dotadas de una función. Estas últimas, son por lo tanto, conocidas como herramientas didácticas, según Dolz y Gagnon (2010, p. 508).

Para definir dichas herramientas, reproducimos la afirmación de Plane y Schneuwly (2000), apud Dolz \& Gagnon (2010, p. 507) que las conciben como "un artefacto introducido en la clase que sirve a las nociones de enseñanza aprendizaje y a las capacidades puestas al servicio de una enseñanza o un aprendizaje en particular."

Es con relación a este "artefacto" que centramos nuestra discusión en este artículo. Nuestro objetivo es demostrar que como profesores, podemos producir también nuestras herramientas didácticas (Dolz \& Gagnon, 2010) al darnos cuenta de que los materiales propuestos para la enseñanza-aprendizaje de una lengua no son suficientes para el programa de enseñanza de un curso.

A este respecto, tenemos en Brasil, varias contribuciones de profesores investigadores que orientan a la elaboración de secuencias de trabajo como herramientas didácticas para las clases. 
Entre ellos ponemos de relieve, en el sur de Brasil, los trabajos de Cristovão (2001, 2004) y E.L.Nascimento $(2004,2005,2009$, 2011).

Cristovão (2001), en su tesis de Doctorado, elaboró secuencias de trabajo, conocidas como secuencias didácticas para la enseñanza de lengua inglesa. Las secuencias didácticas son, según Dolz, Noverraz \& Schneuwly (2004) la construcción de los pasos de la enseñanza. Stutz (2012), orientada por Cristovão (2012) también hace una tesina de maestría sobre la secuencias didácticas como instrumentos para la formación de profesores de inglés.

Partiendo de la misma perspectiva de enseñanza, Cristovão y E.L. Nascimento (2004) nos aportan reflexiones sobre el trabajo con modelos didácticos de géneros a partir de los cuales, según la propuesta teórico-metodológica del interaccionismo socio-discursivo, se prepara una secuencia didáctica para el trabajo con lenguas.

En los modelos didácticos que preceden a la elaboración de una secuencia didáctica, hay que tener presente dos ejes fundamentales, a partir de los cuales se distribuyen los contenidos: el uso del lenguaje (la gramática) y la reflexión.

E.L.Nascimento (2004) presenta un riguroso análisis del género anuncio publicitario institucional, a partir del cual presenta ítems que "permiten vislumbrar las características del género para la construcción de un modelo didáctico" (p. 179).

Otro trabajo de importante referencia producido por E.L.Nascimento (2005) es el que presenta elementos para la elaboración de una secuencia didáctica para el desarrollo de la oralidad, en el dominio del argumentar, a partir de datos obtenidos en clases de portugués ministradas por una profesora de la enseñanza primaria en el norte del Paraná.

En 2011, la autora asocia las secuencias didácticas con los gestos didácticos específicos en la enseñanza-aprendizaje de lengua portuguesa. Se refiere a los modos de proceder, de actuar de una profesora, a partir de una secuencia didáctica. Eso demuestra que la secuencia didáctica o ese tipo de herramienta didáctica resulta bastante útil cuando se tiene presente el proceso de enseñar y aprender una lengua.

Para comprobar eso, en la lengua portuguesa, E.L.Nascimento e Hila (2012) aportan un modelo de secuencia didáctica para trabajar en la enseñanza primaria con el artículo de opinión. Estas autoras sitúan las secuencias como alternativas para proyectos de enseñanza.

Además de estos trabajos, hay varios otros que demuestran la importancia de la producción de herramientas didácticas o secuencias didácticas relacionadas con los textos que reflejan las actividades sociales de los aprendientes (E.L.Nascimento, 2009; Gonçalves; 2009; Saito, 2009), los cuales no describiremos aquí, debido a la extensión de este artículo.

Revista de Gestão e Secretariado - GeSec, São Paulo, v. 4, n. 2, p 73-89, jul./dez. 2013. 
Por lo tanto, apoyándonos en Cristovão y E.L.Nascimento (2004, p.19) que proponen una enseñanza de lenguas basada en la enseñanza sistemática de las capacidades de lenguaje que los géneros textuales envuelven, haremos en el tópico siguiente una descripción del procedimiento metodológico de trabajo que adoptamos, demostrando si los manuales didácticos de español para secretariado proponen lo que constituyen necesidades de aprendizaje en el contexto secretarial internacional.

Basados mayormente en Bronckart (2004), fundador del interaccionismo socio-discursivo, psicólogo de formación, cuya preocupación ha radicado en la didáctica de lenguas, que concibe el lenguaje como una acción mediadora de otras acciones humanas, razonaremos sobre posibilidades de trabajo con textos de la comunicación internacional, específicamente con dos géneros formulaicos (E.P.Nascimento, 2010), a saber, la Carta de Porte y el Certificado de Origen como formas de herramientas de enseñanza-aprendizaje, ya que son ellos que hacen posible las situaciones específicas de la interacción verbal y buena parte del trabajo del profesional de secretariado.

El término géneros formulaicos fue acuñado por E.P.Nascimento (2010). El autor estudió los géneros acta y memo y se trata de un trabajo que está vinculado a un proyecto que conduce en la Universidad Federal del Paraíba, cuyo título es: Estudos Semântico-Argumentativos de Gêneros do Discurso: redação escolar e gêneros formulaicos. (ESAGD).

El género formulaico es un género que se concreta por medio de un formulario, o sea, se realiza en un impreso con espacios en blanco para ser rellenados.

La carta de porte es un formulario en que hay espacios para que consten los nombres y las características de las mercancías que se transportan por vía terrestre. En este documento deben estar presentes los precios de las mercancías, cantidades y los requisitos generales presentes en el artículo 768 del código de comercio.

Ya, el certificado de origen es un formulario en que se constan informaciones que sirven para avalar la procedencia de los productos fabricados en el país, además de confirmar que cumplen con los criterios de origen establecidos por los países a donde se va a exportar, con el objetivo de obtener beneficios arancelarios.

Por lo tanto, conocer los rituales de interacción que tienen lugar por medio de estos formularios es sine qua non para el profesional de secretariado.

Revista de Gestão e Secretariado - GeSec, São Paulo, v. 4, n. 2, p 73-89, jul./dez. 2013. 


\section{MATERIALES DIDÁCTICOS Y EVALUACIÓN DE NECESIDADES DEL CONTEXTO}

$\mathrm{Al}$ averiguar los contenidos trabajados en lengua portuguesa en la enseñanza de Secretariado Ejecutivo, llegamos a la conclusión de que los contenidos aportados por los manuales didácticos de español, disponibles en el mercado, presentan géneros epistolares semejantes a los trabajados en lengua portuguesa. Tal hecho es positivo porque está en consonancia con lo que se considera como necesario para el desarrollo de competencias de lenguaje para un profesional de secretariado ejecutivo. Sin embargo, si volvemos la mirada para la lengua española, vemos que se puede hacer más en la enseñanza, o sea, abarcar también la enseñanza y el aprendizaje de géneros que concretan la comunicación internacional.

Si los géneros discursivos, según Bajtín (2005) se funden en contenido temático, estilo y construcción composicional, nada más coherente que el profesor, al trabajar con la enseñanza de lengua en el curso de Secretariado Ejecutivo, trabaje también con las huellas de cada una de esas dimensiones que concretan los géneros de la comunicación empresarial e internacional, una vez que el profesional de secretariado que trabaja en una empresa multinacional necesita no solo conocer las características de los géneros típicos de la comunicación empresarial - conocidos como los géneros oficiales y comerciales -, sino también los géneros específicos que permiten las transacciones comerciales a nivel internacional. Así, se puede destacar - en las actividades didácticas para secretarios ejecutivos - las huellas de posiciones enunciativas en textos empresariales, tipos de secuencias textuales y aspectos de dialogismo, una vez que en todo enunciado hay una orientación hacia el otro. Además de eso, también se puede trabajar con los dos ejes propuestos por Dolz y Schneuwly (1998) - el eje del lenguaje y de la reflexión - cuando trabajamos con géneros formulaicos de la comunicación internacional.

Siendo los enunciados para Bajtín (2005), la materialización de la utilización de la lengua y en el caso de los géneros de la comunicación internacional, el enunciado se funda sobre representaciones acerca de la cultura del otro, puesto que el mundo social y socio-subjetivo que configuran el contexto de producción de los enunciados ni siempre se comparten entre secretarios y destinatarios de los textos de la esfera internacional, urge la necesidad de ahondar en enfoques de enseñanza que pongan secretarios en formación en contacto con enunciados que posibilitan la comunicación empresarial/internacional de brasileños e hispanoamericanos.

Fundamentándonos en esto, hicimos una investigación bibliográfica, en búsqueda de encontrar manuales didácticos que trabajasen con los géneros de la comunicación internacional.

Revista de Gestão e Secretariado - GeSec, São Paulo, v. 4, n. 2, p 73-89, jul./dez. 2013. 
En el primer momento, la investigación se basó en escudriñar los géneros presentes en siete manuales, los cuales son: I) Cultura y negocios, publicado en 2003 por la Editorial Edinumen; II) Negócios - Manual de español profesional, publicado en 2005 por la Universidad de Alcalá; III) Entorno empresarial, publicado en 2008 por la Edelsa; IV) Español Profesional: Secretariado, publicado por la Eduel; V)En equipo. es, publicado en 2008 por la Edinumen; VI) Empresas Siglo XXI, publicado en 2009 por la Edinumen; VII)Temas de empresa, publicado en 2010 también por la Editorial Edinumen.

Haciendo un recorte para un análisis más amplio, optamos por averiguar si los géneros Carta de Porte y Certificado de Origen estaban presentes tan solo en los libros "Entorno empresarial, En equipo es; Español Profesional: Secretariado y Cultura y Negocios.

Esta nuestra opción metodológica se debe al hecho de que hemos trabajado con los dos primeros manuales en el curso de Secretariado Ejecutivo y con el hecho de los dos últimos tratarse de manuales destinados a la enseñanza de español, centrada en el aprendiente latinoamericano.

En cuanto a los géneros de textos presentes en los manuales, están presentes en el manual Entorno empresarial: 4 artículos de opinión, 3 artículos expositivos y 1 artículo publicitario.

Ya, en el manual En equipo.es, los géneros presentes son memos, invitaciones, convocatoria de reunión, comunicado interno, carta de felicitación, carta de comunicación de pago, carta de reclamación e invitación.

En el manual Español Profesional: Secretariado, los géneros relacionados con el área son: currículo, anuncios de trabajo, carta de presentación, cartas comerciales, cartas de solicitación, cartas de ofertas de productos, carta publicitaria, e-mail, artículo expositivo e instrucciones para entrevista de trabajo. Estos géneros demuestran que la organización de los manuales está vuelta hacia la carrera profesional de secretario/a.

En el manual Cultura y negocios: en cada unidad hay un artículo sobre la cuestión económica de un país latinoamericano. Hay también una noticia relacionada con la economía en América.

En cuanto a la razón por la cual no hemos encontrado los géneros "Carta de Porte" y “Certificado de Origen” en los manuales Entorno empresarial y En equipo.es puede deberse al hecho de que los manuales reproducen un conjunto de valores estereotipados con relación a la enseñanza de español en el curso de Secretariado y en su proyecto de enseñanza no se consideran contextos de producción específicos como el del profesional de secretariado brasileño que puede ejercer actividades de asesoría, en las cuales va a necesitar utilizar una lengua que sufre 
variaciones, según el país en que se utiliza, una vez que la lengua está asociada a la cultura y, en este caso, hay que tener presente que al hablar de español, se habla también del binomio lengua/cultura de 21 países diferentes.

Eso no significa decir que el enfoque de los manuales deba ser las informaciones relacionadas con los 21 países porque sabemos que esto sería casi imposible hacerse en un único manual; sin embargo, nos intriga el hecho de que ninguno de ellos centra la atención a formularios de negociación entre los 3países que mantienen relaciones más arraigadas con Brasil, o sea, nos referimos a Argentina, Uruguay y Paraguay.

En cuanto al libro Español Profesional: Secretariado fue elaborado específicamente para el alumno brasileño; no obstante, la razón por la que no encontramos géneros de la comunicación internacional puede estar asociada al hecho de ser todavía un material para el nivel básico de enseñanza.

Por otra parte, en el manual Cultura y Negocios, los géneros textuales tienen el objetivo de informar sobre la cuestión política y económica de algunos países latinoamericanos. No obstante, aún estando el Mercosur inserto en la cuestión económica, no se hace mención a los géneros "Carta de Porte" y "Certificado de Origen".

Nuestra justificativa en cuanto a la escoja por analizar los manuales didácticos se debe al hecho de que resuenan en los pasillos de nuestro departamento de Letras de la Universidad las consideraciones de muchos profesores de que el trabajo en el salón de clase sin ese soporte didáctico es dificultoso. Sin embargo, en el transcurrir de nuestras clases, sentimos la necesidad de realizar actividades más condichas con las necesidades de los alumnos, lo que implicó presuponer la funcionalidad social y contextual de nuestro trabajo con los textos en el salón de aula.

Para tanto, adoptamos los principios del interaccionismo socio-discursivo para incrementar la enseñanza de español en el curso de Secretariado de modo que pudiéramos, de acuerdo con Dolz y Schneuwly (2004) hacer posible a los secretarios en formación el desarrollo de capacidades de lenguaje para actuar en su ambiente laboral con los géneros seleccionados para la enseñanza y aprendizaje.

Así, pusimos en marcha el proceso de búsqueda en los medios sociales del género para hacer el modelo didáctico y la transposición del mismo en forma de secuencia didáctica para el aula.

Vale resaltar que, al poner en acción el trabajo con dichos contenidos que consideramos enseñables en nuestro contexto de enseñanza y aprendizaje, hicimos a la vez una "transformación del objeto de enseñanza" (E.L.Nascimento, 2011, p. 421). Dicha transformación suele suceder porque al construir modelos didácticos de géneros (Dolz y Schneuwly, 2004) se extraen las

Revista de Gestão e Secretariado - GeSec, São Paulo, v. 4, n. 2, p 73-89, jul./dez. 2013. 
características enseñables en un determinado contexto, en que el profesor se basa en la pertinencia y adaptación al contexto de producción y recepción del texto y a las capacidades de los alumnos.

Los modelos didácticos sirven para la identificación de las dimensiones enseñables de un género textual, generando actividades y secuencias de enseñanza. Con el modelo, según Dolz \& Gagnon, 2010, p.511) se pueden integrar nuevos textos con los preconstructos ya existentes.

De este modo, lo que deseamos demostrar, al proponer el trabajo a partir de la perspectiva del interaccionismo socio-discursivo es la transformación del texto real que puede promover el docente, al llevarlo a sus alumnos con el objetivo de desarrollar capacidades de lenguaje por medio de los textos. Lo ejemplificaremos en el tópico que sigue por medio de las actividades que forman parte de una secuencia didáctica que preparamos con los géneros "Carta de porte" y "Certificado de origen" que pueden ser considerados géneros textuales que reflejan prácticas sociales de referencia.

\section{SOBRE EL TRABAJO CON LOS GÉNEROS Y EL DESARROLLO DE CAPACIDADES DE LENGUAJE}

La noción de capacidades de lenguaje, acuñada por Dolz, Pasquier y Bronckart (1993, apud Dolz y Schneuwly, 1999) se ha extendido a la enseñanza de lenguas en general. Empezó con el trabajo desarrollado por medio de secuencias didácticas en francés por los fundadores del interaccionismo socio-discursivo (Dolz, Pasquier \& Bronckart, 1993, apud Dolz \& Schneuwly, 1999); Dolz y Schneuwly (2004), y sigue siendo empleada en el trabajo con los géneros de textos, tanto en lengua portuguesa como en lengua inglesa y en lengua española. En cuanto a esta última lengua, Dora Riestra (2008), autora argentina defiende las consignas de trabajo en que se desarrollen capacidades de lenguaje.

De acuerdo con Dolz, Pasquier y Bronckart (1993), al trabajar con los géneros textuales en el aula, es preciso no sólo movilizar las representaciones sobre el medio físico de la interacción comunicativa y sobre el contexto de producción (capacidad de acción - de ahora en adelante CA); como también es necesario también observar cómo es organizada la infraestructura del texto, o sea, los tipos de secuencias y tipos de discurso (capacidades discursivas - de ahora en adelante CD). Además de eso, hay que analizar los mecanismos de textualización y enunciativos - que tienen que ver con la distribución de las voces y de expresión de modalizadores, además de volver la mirada 
hacia la construcción de enunciados e ítems lexicales (capacidades lingüístico/discursivas, de ahora en adelante CLD).

Eso significa que hablar en capacidades de lenguaje es referirse al modo como los alumnos interaccionan con un texto, mirando su contexto intratextual y extratextual, asícomo su estilo composicional, sus voces y sus usos lingüísticos/discursivos.

De ese modo, desarrollar capacidades es sinónimo de apropiarse de los esquemas de utilización del un género de texto y por eso, dichos esquemas necesitan ser trabajados por medio de las actividades propuestas en el material didáctico utilizado para la enseñanza.

Desde este encuadre teórico, pasaremos a demostrar una de las actividades que desarrollamos, fijándonos en las capacidades que pretendíamos desarrollar en los alumnos.

Antes, queremos aclarar que organizamos las actividades con los géneros en dos estadios: I) presentamos la situación de enseñanza y aprendizaje en que hicimos el sondeo en clase con relación al conocimiento de los alumnos sobre el género; II) construimos el sentido del trabajo con los géneros Carta de Porte y Certificado de Origen.

La primera actividad consistió en entregar el formulario referente al género Carta de Porte para que los alumnos rellenaran con el intento de identificar el género y para averiguar si tendrían dudas para rellenarlo.

Optamos por ese plan de enseñanza y aprendizaje porque la propuesta de Dolz, Noverraz y Schneuwly (2004, p. 95-128) es la de comenzar con una producción inicial para levantar las capacidades de los alumnos con el fin de actuar por medio del género. Para tanto, excluimos del formulario el nombre del género para no interferir en el resultado de la producción de los alumnos.

Como resultado, los alumnos no supieron decir de que género se trataba y manifestaron en ese momento, algunas dificultades con relación al léxico. Por lo tanto, las palabras que generaron dudas fueron trabajadas posteriormente y correlacionadas con las capacidades de lenguaje que serán expuestas en la tabla 1 que presentamos en el cuerpo de este artículo.

Así, para demostrar como procedemos en el aula, demostraremos la actividad 2 de la secuencia didáctica que elaboramos, en la cual explicamos que se trata de formularios relacionados con las situaciones de exportación/ importación y posteriormente la tabla 1 en que relacionamos las tareas con el desarrollo de las capacidades de lenguaje.

Revista de Gestão e Secretariado - GeSec, São Paulo, v. 4, n. 2, p 73-89, jul./dez. 2013. 


\section{Actividad 2:}

\section{Una mirada hacia los géneros textuales del área de exportación/importación}

El amplio desarrollo tecnológico que ha ocurrido desde la década de 80 ha transformado el trabajo del profesional de secretariado, volviéndolo más dinámico y a la vez más complejo, ya que nuevas formas de interactuación se han manifestado. Entre ellas, es de fundamental importancia el papel de las/os secretarias/os que trabajan en empresas exportadoras o importadoras.

El papel que asume el profesional de empresas como éstas es de un incansable aprendiente debido a las situaciones diversas de interactuación intercultural que concretizan las negociaciones entre las empresas.

Teniendo presente la necesidad del desarrollo del conocimiento con relación al transporte de mercancías de países del Mercosur, elija los tipos de documentos que desde su punto de vista deben formar parte del conocimiento de secretarios para efectuar las negociaciones:

( ) Lista de empaque

( ) factura comercial

( ) Guía de Transporte

( ) Multinota

( ) Carta de Porte

( ) Conocimiento de Embarque

( ) Hoja de Ruta

( ) Certificado de Origen

Esta actividad fue elaborada para despertar el interés de los alumnos por el aprendizaje de otros géneros textuales que forman parte de ese ámbito de la comunicación de exportación/importación.

El objetivo fue demostrar que el formulario que estaban rellenando sobre el conocimiento de transporte, debería ir acompañado de, en lo mínimo, más dos formularios que son la factura comercial y el certificado de origen del producto que sería exportado para que el importador pudiera tener la reducción o la exención del impuesto de Importación.

De ese modo, el alumno estaría movilizando su capacidad de acción para comprender el

Revista de Gestão e Secretariado - GeSec, São Paulo, v. 4, n. 2, p 73-89, jul./dez. 2013. 
contexto de producción de la elaboración de los tres géneros textuales, una vez que uno depende del otro.

Tras ese sondeo, les entregamos tres formularios para que observaran las diferencias de cada uno y para que los nombraran. Observaron que la diferencia entre la Carta de Porte y el Certificado de Origen es que el léxico del primero es diferente del léxico del segundo género, ya que poseen términos como flete, reembolso contra entrega y transportadora.

Otro aspecto que les llamó la atención es que en el reverso del Certificado de Origen, había modalizadores lógicos como: no podrá presentar tachaduras, correcciones o enmiendas; deberá ser emitido a partir de la fecha de emisión de la factura comercial, etc.

Esa actividad, por lo tanto, tuvo como objetivo no solo el sondeo del conocimiento de los académicos de Secretariado, sino también desarrollar capacidades de acción, visto que encontrarían en los géneros los términos como "Cámara de Industrias del Uruguay" y tendrían que reflexionar sobre el mundo físico y sociopolítico en que el formulario estaría circulando.

La misma actividad pudo, a la vez, proporcionar el desarrollo de la capacidad discursiva porque los alumnos tuvieron que analizar la estructura composicional del formulario. Eso les ha permitido enterarse de que predomina la descripción de informaciones y la prescripción en el reverso del certificado de origen.

Tras ese contacto inicial con los formularios, hicimos las demás actividades, las cuales se organizaron en torno de cuestionamientos sobre los géneros estudiados. Para ejemplificar las capacidades de lenguaje que quisimos desarrollar con las actividades de la secuencia didáctica, organizamos la tabla que sigue, exponiendo al lado izquierdo de la tabla 1 algunas de las preguntas que hicimos y al lado derecho, las capacidades que visaban a desarrollar. 


\begin{tabular}{|c|c|c|c|}
\hline PREGUNTAS & CA & CD & CLD \\
\hline $\begin{array}{l}\text { a) ¿Qué tipo de empresas podrá exigir que el profesional de Secretariado sepa trabajar } \\
\text { con los géneros Certificado de Origen, Carta de Porte y Factura comercial? }\end{array}$ & X & & \\
\hline b)¿Quiénes son los responsables en la empresa por rellenar el Certificado de Origen? & $\mathrm{X}$ & & \\
\hline c) ¿Cuál es el primer procedimiento relativo al certificado de origen? & $\mathrm{X}$ & & \\
\hline d) ¿Con cuál nomenclatura fue desarrollado el Certificado de Origen? & & & $\mathrm{X}$ \\
\hline $\begin{array}{l}\text { e)En lo que se refiere al conocimiento de transporte, según Barbosa y Martins (2002) } \\
\text { hubo una evolución en el número de empresas y flotas autorizadas en el transporte por } \\
\text { carretera en el Mercosur, si comparamos con los años de } 1990 \text { a } 2000 \text {. De } 36 \\
\text { empresas, fueron autorizadas } 753 \text { y de } 1807 \text { flotas, fueron autorizadas } 35.113 \text { flotas en } \\
\text { Brasil. ¿A qué se debe eso? }\end{array}$ & $X$ & & \\
\hline f) ¿Cómo se rige el Transporte Internacional por carreteras en el Cono Sur? & $\mathrm{X}$ & & \\
\hline g) ¿Cuál es el significado de FOB? & & & $\mathrm{X}$ \\
\hline h) ¿ Qué significa CIF? & & & $\mathrm{X}$ \\
\hline i) ¿Cuál es el significado de consignatario? & & & $\mathrm{X}$ \\
\hline $\begin{array}{l}\text { j) ¿Dónde se indica el número de mercancías en el Certificado de Origen? ¿Podrán } \\
\text { exceder } 20 \text { mercancías? }\end{array}$ & & & $X$ \\
\hline k)¿Cuándo se indica "a granel” en el Certificado de Origen? & & & $\mathrm{X}$ \\
\hline 1) ¿Qué significa NCM? & & & $\mathrm{X}$ \\
\hline
\end{tabular}

Delante de lo expuesto en la tabla, se puede observar que para que los alumnos consiguieran responder a la pregunta $a$, necesitarían movilizar sus conocimientos en relación con los tipos de empresas y reflexionar sobre la clasificación de la empresa, es decir, si se clasifica en la actividad de fabricante, fabricantes y exportadoras o solamente exportadora o prestadora de servicios.

Se trata de una actividad en que el académico precisaría movilizar su capacidad de acción

Revista de Gestão e Secretariado - GeSec, São Paulo, v. 4, n. 2, p 73-89, jul./dez. 2013. 
para contestarla.

Para contestar a la pregunta b, el alumno necesitaría saber que en la empresa, únicamente el administrador podrá catastrar el responsable por rellenar el Certificado de Origen. Eso significa que es un documento que podrá ser rellenado por el administrador y generalmente por su asesor/a ejecutivo/a o secretario/a que ocupa cargos en empresa de gran porte. Tal hecho demuestra la importancia de una secretaria apropiarse del género para saber emplearlo, caso el administrador de la empresa la catastre para rellenar ese género.

Para contestar a la pregunta $c$, el profesional en formación de Secretariado necesitaría desenvolver su capacidad de acción, o sea, una capacidad relacionada con ese mundo físico y sociosubjetivo, visto que la respuesta es que el primer procedimiento es el catastro de mercancías de la empresa. En ese catastro, registrará su código interno y el nombre comercial de la mercancía, que serán utilizados para generar la factura comercial.

En la pregunta $d$, los alumnos necesitarían movilizar su capacidad lingüístico -discursiva para decir que fue desarrollado con base en la NCM - Nomenclatura Común del Mercosur y su correlación Naladi. (ALADI) Asociación Latinoamericana de Integración.

La pregunta e moviliza conocimientos de mundo sobre la cuestión de transportes en la exportación/importación. Los académicos, por lo tanto, necesitarían movilizar su conocimiento de mundo relacionado con las carreteras entre Brasil y los países del Mercosur para contestar a esa pregunta.

Eso refuerza la necesidad de que un profesional de secretariado conozca muy bien la lengua y los géneros que concretan las acciones de importación y exportación, visto que es un sector en expansión.

Para contestar a la pregunta $f$, el alumno necesita movilizar su capacidad de acción para decir que el Transporte Internacional por carreteras en el Cono Sur se rige por el Acuerdo sobre Transporte Internacional Terrestre (ATIT) o para decir que no sabe. En este caso necesitaría, por lo tanto, apropiarse de esos conocimientos para actuar como asesor ejecutivo de una empresa de gran porte.

Para contestar a las preguntas $g$ y $h$, el alumno necesita tener un conocimiento lingüísticodiscursivo, o sea, conocer el significado de los términos. Necesita saber que se trata de tipos de fletes y que FOB (Free On Board) significa flete pagado por el comprador y CIF(Cost Insurance Freight) se refieren a los costes, seguro, flete y desembarazos legales que son pagados por quien provee la mercancía.

Revista de Gestão e Secretariado - GeSec, São Paulo, v. 4, n. 2, p 73-89, jul./dez. 2013. 
Para contestar a la pregunta $i$, el aluno necesita saber que consignatario es la persona natural o jurídica para quien se destina la mercancía.

Para contestar a la pregunta $j$, los alumnos necesitan movilizar las capacidades discursivas para encontrar en el formulario el lugar en que pueden insertar el número de productos y las capacidades lingüístico-discursivas para entender el vocabulario referente a esas informaciones.

Para contestar a la pregunta $K$, el alumno necesita movilizar la capacidad lingüísticodiscursiva porque la expresión “a granel” se utiliza cuando las mercancías no están empaquetadas.

El alumno necesita tener solo esta capacidad para contestar a esa pregunta porque precisa conocer la nomenclatura que significa Nomenclatura Común del Mercosur.

En resumen, con base en lo descrito en la actividades que presentamos en ese artículo, se puede constatar que fueron trabajadas más las capacidades de acción y lingüístico-discursivas. Eso porque las capacidades de acción son esenciales para este profesional, una vez que un secretario/a necesita conocer aspectos relacionados con el mundo físico, social y subjetivo del género de texto por medio del cual realiza las negociaciones. Además de esto, este profesional, no puede prescindir de las capacidades lingüístico-discursivas para poder leer y completar los formularios.

En cuanto a las capacidades discursivas, estas casi no fueron trabajadas porque la organización estructural del formulario ya viene patronizada y por lo tanto, no hay como la/el secretaria/o utilizar una estructura diferente de la que es patrón.

\section{ALGUNAS CONSIDERACIONES FINALES}

El contexto de trabajo de los graduandos en Secretariado es bastante heterogéneo. Actualmente los/las secretarias/os ejecutivas/os tienen la posibilidad de trabajar en plazas de secretaria/o o asistencia ejecutiva que es un tipo de secretaria/o de dirección. Esa posibilidad hay tanto en empresas nacionales como multinacionales.

Por esa razón, nuestro enfoque en ese trabajo fueron los géneros textuales que el profesional de secretariado ejecutivo podrá ejecutar en su realidad profesional, movilizando para eso, los recursos de la comunicación internacional cristalizado y sedimentado en el área de exportación/ importación. 


\section{REFERENCIAS}

Bajtín, M. (2005). Estética de la creación verbal. Buenos Aires: Siglo XXI Editores Argentina.

Bronckart, J. P. (2004). Actividad verbal, textos y discursos: por un interaccionismo sociodiscursivo.(V. de. Salvador, \& M.J. Carrión, Trad.)Fundación Infancia y Aprendizaje. Recuperado em 13 março, 2013, de http://books.google.com.br/books?id=Rd1vnZvZ6WgC\&printsec=frontcover\&hl=pt$\underline{B R \# v=\text { onepage } \& q \& \mathrm{f}=\text { false }}$.

Cristovão, V. L. L., \& Nascimento, E. L.(orgs). (2004). Gêneros textuais: teoria e prática (Vol.1, pp. 18 - 31) Londrina: Moriá.

Cristovão, V. L. L. (2001). Gêneros e ensino de leitura em LE: Os modelos didáticos de gêneros na construção e avaliação de manual didático. Tese de Doutorado, Linguística Aplicada e Estudos da Linguagem, Pontifícia Universidade Católica de São Paulo, São Paulo, Brasil.

Dolz, J., Pasquier, A \& Bronckart, J. P. (1993). L`acquisition des discours émergence d`une compétence ou apprentissage de capacités langagier`es? Études de Linguistique Appliquée, (n.92), 23-37.

Dolz, J., \& Schneuwly, B. (1998). Pour un enseignement de l oral. Initiation aux genres formels àl'école. Paris, ESF Editeur.

Dolz, J., \& Schneuuwly, B. (1999). Os Gêneros escolares. (G. Sales Cordeiro, Trad.) Revista Brasileira de Educação,11, pp.5-16.

Dolz, J., Noverraz, M., \& Schneuwly, B.(2004). Seqüências didáticas para o oral e a escrita: apresentação de um procedimento. In:B. Schneuwly \& J. Dolz. Gêneros orais e escritos na escola. Campinas: Mercado de Letras.

Dolz, J., \& Schneuwly, B. (2004).Gêneros orais e escritos na escola. (G. Sales Cordeiro \& R.Rojo,Trad.) Campinas: Mercado de Letras.

Gagnon, R, Dolz, J. (2010). El género textual, una herramienta didáctica para desarrollar el lenguaje oral y escrito.(V. Sanchez, Trad.) Lenguaje, 38 (2),497-527.

Gonçalves, A.V. (2009). Ferramentas didáticas e ensino: da teoría à prática de sala de aula. In: E.L. Nascimento (Org.)Gêneros Textuais: da didática das línguas aos objetos de ensino.São Carlos, Editora Claraluz.

Nascimento, E.P. (2010). Gêneros do Universo Oficial/Empresarial: Para Além dos Manuais de 
Redação. Revista de Gestão e Secretariado. 1(2),123-142. Recuperado em 15 março, 2013 de http://revistagesec.org.br/ojs-2.2.4/index.php/secretariado/article/view/21/85

Nascimento, E. L. (2005). Gêneros em expressão oral; elementos para uma sequiência didática no domínio do argumentar. Signum: Estudos da Linguagem. Revista do Programa de PósGraduação em Estudos da Linguagem. 8(1), 131-156.

Nascimento, E. L. (2009). Gêneros da atividade, gêneros textuais: Repensando a interação em sala de aula. In: E.L. Nascimento (Org.). Gêneros Textuais: da didática das línguas aos objetos de ensino. São Carlos, Editora Claraluz.

Nascimento, E. L. (2011). A dupla semiotização dos objetos de ensino-aprendizagem: dos gestos didáticos fundadores aos gestos didáticos específicos. Signum: Estudos Linguísticos, 14(1), 421445.

Nascimento, E. L, Hila, C.V.D. (2012). Práticas de Sala de Aula: as sequências didáticas como ferramentas de ensino. In: A. Gonçalves, \& M.R. Petroni (Orgs.) Formação inicial e continuada de professores: o múltiplo e o complexo nas práticas educativas(pp.135-167). Dourados: Ed. UFGD.

Riestra, D.(2008). Las consignas de la enseñanza de la lengua: un instrumento didáctico. Novedades Educativas, 211, 14 -22.

Saito, C.L.N. (2009) Telejornal: um gênero pra o letramento midiático. In: E.L. Nascimento. (Org.)Gêneros Textuais: da didática das línguas aos objetos de ensino (195-224). São Carlos, Editora Claraluz.

Stutz, L. (2012) Sequências didáticas, socialização de diários e autoconfrontação: instrumentos para a formação inicial de inglês. Tese de Doutorado em Estudos da Linguagem, Universidade Estadual de Londrina, Londrina, Paraná, Brasil.

Revista de Gestão e Secretariado - GeSec, São Paulo, v. 4, n. 2, p 73-89, jul./dez. 2013. 